\title{
Influence of electrospun nanofibers on the interlaminar properties of unidirectional epoxy
}

resin/glass fiber composite laminates

\author{
Hamed Saghafi ${ }^{1}$, Roberto Palazzetti ${ }^{2,}{ }^{*}$, Andrea Zucchelli $^{1}$, Giangiacomo Minak ${ }^{1}$ \\ ${ }^{1}$ Department of Industrial Engineering (DIN), University of Bologna, Italy \\ ${ }^{2}$ DMEM department, University of Strathclyde, UK
}

*Correspondence author: roberto.palazzetti@strath.ac.uk - Tel: +44 (0) 1415484294

\begin{abstract}
Nylon 6,6 nanofibers were interleaved in the mid-plan of glass fiber/epoxy matrix composite laminates for Mode I and II fracture mechanic tests. The present study investigates the effect of the nanofibers on the laminates' mechanical response. Results showed that Nylon 6,6 nanofibers improved specimen's fracture mechanic behaviour: the initial energy release rates $G_{I C}$ and $G_{\| c}$ increased $62 \%$ and $109 \%$ respectively when nanofibrous interlayer was used. Scanning Electron Microscope (SEM) micrographs showed that nanofiber bridging mechanism enhances performances of the nanomodified specimens, still able to link the layers when the matrix is broken.
\end{abstract}

\section{Keywords}

Electrospinning, laminates, composites, delamination

\section{Introduction}

Laminates' superior properties, compared to traditional materials such as steel and aluminium, led them to be among the widest used composite structures in engineering today, especially for high performance application. Despite their excellent characteristics, due to their ply-to-ply structure, 
the weakness of the interfaces is still an issue for composite's designers and users. Delamination is a discontinuity between adjacent plies and can be viewed as an interface crack between two anisotropic materials: it is an intrinsic and severe problem of laminates that can ultimately undergo structural failure. Delamination can be due to many factors such as interlaminar stresses, stress concentration at free edges, joints, matrix cracks, out-of-plane loading, physical discontinuities or mismatch in physic-mechanical properties (e.g. coefficient of linear thermal expansion) between adjacent layers [1]. The interest of the research community on delamination is highly connected to industry since many companies are facing this problem, which is particularly critical for aerospace and aeronautical applications. So far researchers have developed many techniques to solve the delamination problem such as fiber surface treatments [2], interlayers [3], stitching polymeric filaments [4], optimum stacking sequence [5], through-the-thickness reinforcement [6], Z-pins [7], fiber braiding [8] and nanofibers [9].

The present study is focused on interleaving epoxy resin based composite laminates with nanofibers, following the idea and the patent of Dzenis [10], who added Polybenzimidazole (PBI) nanofibers to ply-to-ply interfaces and registered a significant improvement in fracture toughness of the whole laminate. While reinforcing fracture toughness of composites, it is shown that nanofibrous reinforces with a proper thickness, does not cause any reduction of in-plane mechanical properties of the laminates $[11,12]$, while other methods such as Z-pin and fiber braiding can decrease these properties significantly [13-16].

Since the patent of Dzenis, several papers can be found on composite laminates interleaved with electrospun nanofibers [17-26]: many polymers have been used and tested so far [17-21], and Nylon 6,6 was proved to be the most suitable for the purpose of reinforcing epoxy-based composites, due to its good mechanical properties, processability, and high melting temperature which allows the nanofibers to maintain their morphology during the most common composite's curing processes $[27,28]$. 
In the present study thin Nylon 6,6 nanofibrous sheets have been interleaved into glass fibers/epoxy resin composite laminates with the purpose of investigating the effect of the interleave, and eventually the mechanism the nanofibers play into the interface when the sample is loaded under Mode I and Mode II fracture mechanics.

Shivakumar et al. [22] conducted Mode I fracture, low-velocity impact, and Dynamic Mechanical Analysis (DMA) tests on virgin and Nylon 6,6-modified carbon/epoxy laminates, showing increased mechanical performances of the material with respect to the pristine specimens. Palazzetti et al. $[11,23,24]$ performed an extended experimental campaign on woven carbon/epoxy composites showing the influence of Nylon 6,6 interleave on the fracture behaviour of laminates.

So far, different toughening mechanisms caused by nanofibers have been observed $[17,20,24]$. Zhang et al. [17] investigated the toughening mechanism of a nanointerleave into a carbon/epoxy laminate in Mode I. They showed that fracture toughness was improved only once the polymerisation-induced phase separation between the thermoplastic nanofibre and epoxy matrix occurred in the interlayer. Li et al. [20] interleaved polysulfone (PSF) into carbon/epoxy composites and showed that PSF nanofibers converted to spherical particles during the curing process are able to significantly improve the interlaminar properties of the composites.

Despite many studies on carbon fiber laminates, few have been completed on glass fiber laminates, and their interaction with polymeric nanofibers. Liu et al. [12] applied 150-500 nm Nylon 6 nanofibers to glass/epoxy laminates and showed that the interleave did not affect the mechanical performance of the composite laminates significantly. Sadeghian [25] introduced Carbon NanoFibers (CNF) into glass/polyester composites, showing significant improvement in the $\mathrm{G}_{\mathrm{IC}}$ as $1 \mathrm{wt} \% \mathrm{CNF}$ were added to toughen the resin. Microscopy pictures showed that the fracture surfaces of the $1 \mathrm{wt} \%$ CNF toughened laminates were more complex than the fracture surfaces of regular polyester/glass fiber composites. 
Although the effect of Nylon 6,6 on mechanical behaviour of GFRP laminates was already considered by the authors in [18], a new curing process has been adopted in the present study, in order to improve the benefits of applying nanofibers between composite layers. Scanning Electron Microscope (SEM) micrographs are also used to consider the nanofibers' reinforce mechanism.

\section{Materials and methods}

\section{Nylon 6,6 nanofibers}

Figure 1 (left) illustrates an overview of the electrospinning machine designed and manufactured by Spinbow (San Giorgio di Piano, 40016 Italy), for producing nanofibers used for the experiments. Nanofibers were electrospun horizontally.

Polymeric solution was made by dissolving Nylon 6,6 Zytel E53 NC010, purchased from DuPont in the form of pellets, in formic acid and chloroform, purchased from Sigma Aldrich and used as received. Nylon 6,6 concentration was $14 \%$ in formic acid/chloroform (50:50 v/v) solution. The electrospinning parameters were as follows: 1- applied voltage: $23 \mathrm{kV}, 2$ - needles inner diameter: $0.8 \mathrm{~mm}, 3-$ needles-collector distance: $10 \mathrm{~cm}, 4-$ feed rate: $0.005 \mathrm{ml} / \mathrm{min}$ per nozzle, 5environment: humidity at $40 \%$ and room temperature. The electrospinning duration was about 60 minutes and final mat thickness was $25 \pm 8 \mu \mathrm{m}$. An SEM image of the electrospun nanofibers is shown in Figure 1 (right). The diameters of nanofibers were $150 \pm 15 \mathrm{~nm}$. The fiber diameter distribution was determined by measuring 200 fibers per sample, with image acquisition software (EDAX Genesis). 

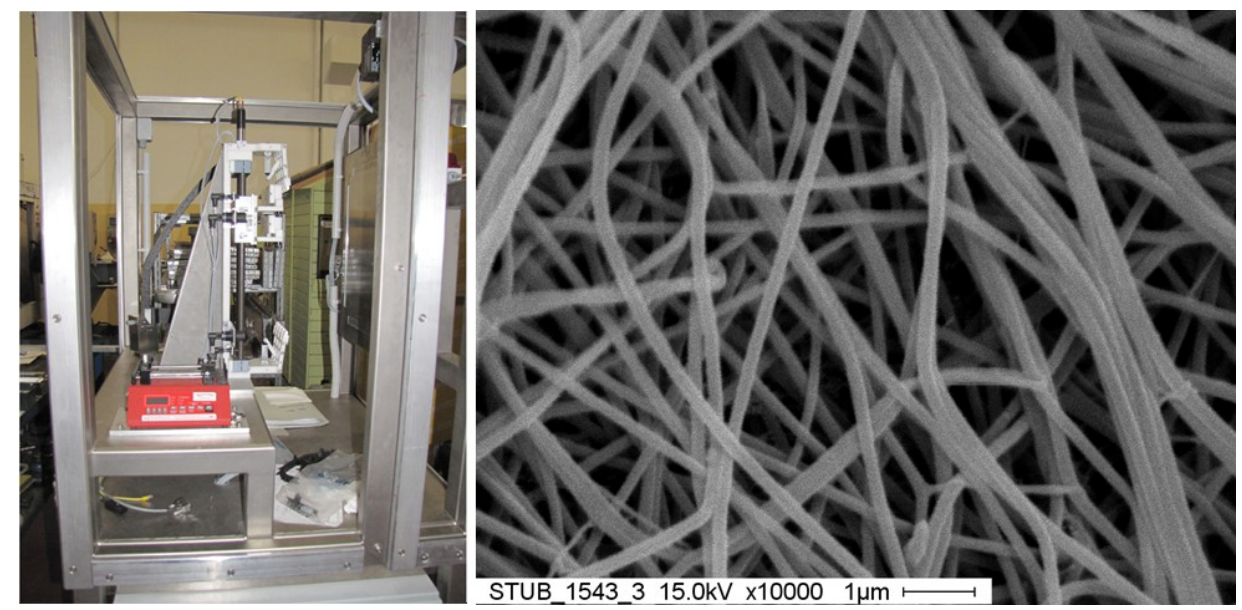

Figure 1. Electrospinning machine (left) and Nylon 6,6 nanofibers (right).

Thermal properties of the electrospun Nylon 6,6 mat were investigated by means of differential scanning calorimetry using a TA Instruments Q100 DSC equipped with the LNCS low-temperature accessory. The Nylon 6,6 nanofibrous mat was found to be particularly suitable for the purpose of the present work due to its chemical and physical characteristics; it shows excellent adhesion to epoxy matrix, as shown in Figure 1 of [23] and Figure $4 \mathrm{f}$ in [28], and it is characterized by a highmelting crystal phase $\left(T_{m}=263^{\circ} \mathrm{C}\right.$ at $10^{\circ} \mathrm{C} / \mathrm{min}$; DAM; ISO $11357-1 /-3, \Delta H_{m} 65 \mathrm{~J} / \mathrm{g}$, by DSC). Therefore it was observed that the prepreg curing treatment at a temperature $\left(130^{\circ} \mathrm{C}\right)$ well below the Nylon 6,6 melting temperature did not cause any modification in electrospun mat shape or in Nylon 6,6 fiber morphology.

After the electrospinning, the nanofibrous mat was kept under vacuum over $\mathrm{P}_{2} \mathrm{O}_{5}$ at room temperature overnight to remove residual solvents and avoid moisture absorption. The presence of water possibly released by the fibers into the epoxy matrix, may interfere with the curing process, detrimentally affect both the thermal and mechanical properties of the composites and accelerate ageing-related degradation phenomena.

Mass per unit area of the nanofibrous mat has been estimated in $25 \mathrm{~g} / \mathrm{m}^{2}$ by averaging the weight of 10 large nanofibrous sheets. 


\section{Laminate Fabrication}

Unidirectional glass/epoxy prepreg 1017 from Angeloni (Italy) was used as composite material. Areal density of the fibers is $430 \pm 2 \mathrm{~g} / \mathrm{m}^{2}$ and matrix content of the laminate is $35 \%$ in volume. Double Cantilever Beam (DCB) and End Notched Flexure (ENF) specimens were prepared for tests under Mode I and Mode II loading respectively, by stacking 10 layers of material, $20 \mathrm{~mm}$ wide. Mode I and Mode II specimens were $125 \mathrm{~mm}$ and $150 \mathrm{~mm}$ long respectively. Samples were cut from a large lamina by using a diamond rotating saw. For each configuration 3 specimens were manufactured and tested. Delamination was initiated by laying down a $15 \mu \mathrm{m}$ thick Teflon sheet in the mid-interface, as shown in Figure 2; crack length was $60 \mathrm{~mm}$ and $70 \mathrm{~mm}$ long for Mode I and Mode II specimens respectively. DCB specimens were prepared by gluing aluminium tabs on the delaminated end of the specimens (see Figure 2 left).
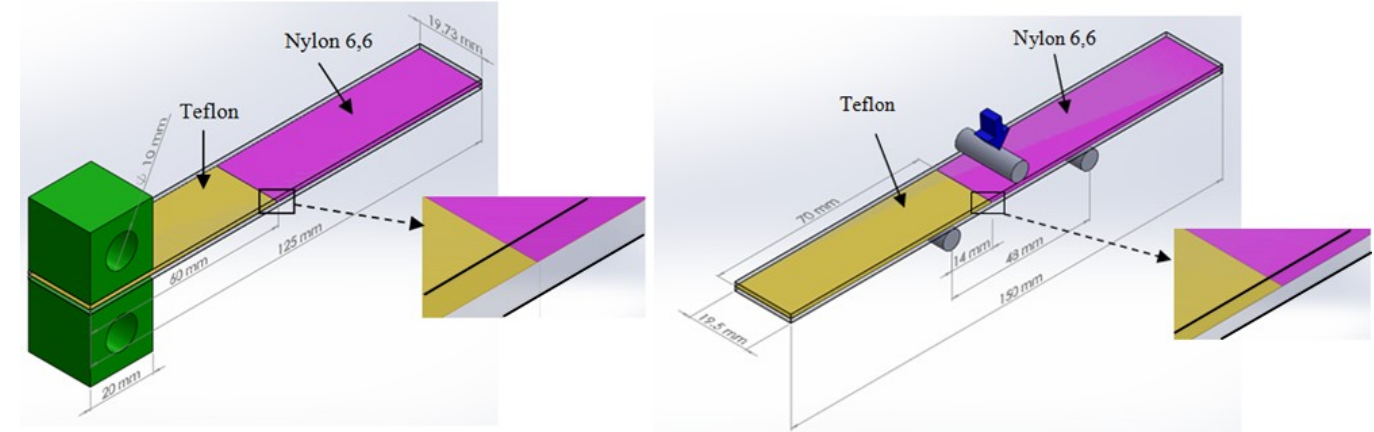

Figure 2. Specimens for: left) double cantilever beam tests; right) end notch flexure tests.

Nanomodified configurations were obtained by laying down a nanofibrous sheet in the midinterface of the specimens during the lay-up. DCB and ENF samples have an interlayer surface of 2466 and $2925 \mathrm{~mm}^{2}$ respectively, thus the weight of the nano-interlayer is estimated to be 0.061 and $0.073 \mathrm{~g}$ per specimen (DCB and ENF respectively), which can be considered negligible. No thickness differences have been observed for both nanomodified DCB and ENF specimens compared to virgin ones; in [24] it has already shown that a thin nanointerleave like those here 
used does not increase specimen's thickness, due to the very high porosity of the mat and the pressure applied during the curing process.

Specimens were kept overnight in the autoclave under 6 bars of pressure and at room temperature, before starting the curing process the day after, following supplier's instructions [11] In previous works of some of the authors $[11,26]$, the specimens were cured right after the manufacturing process; it is because of leaving the interleaved specimens overnight at high pressure which leads to improve the embedding of the fibers, reduces void content in the interface, and eventually increases the Mode I and Mode II fracture toughness significantly.

\section{Fracture tests}

Mode I and Mode II fracture tests were conducted using a computer-controlled servo-hydraulic universal testing machine Instron 8033 with 1 and $5 \mathrm{kN}$ load cells for DCB and ENF tests respectively. The cross-head speed was controlled at $1.5 \mathrm{~mm} / \mathrm{min}$ and data was recorded 10 times per second. The initial Mode I critical strain energy release rate $\left(G_{I C}\right)$ was obtained from the beam theory presented in the ASTM D5528 [29]:

$$
\mathrm{G}_{\mathrm{IC}}=\frac{3 \mathrm{P} \delta}{2 \mathrm{Ba}}
$$

where $\mathrm{P}$ is the maximum load, $\delta$ is the displacement; $\mathrm{B}$ and $a$ are the specimen width and the crack length respectively.

Critical strain energy release rate for Mode II fracture $\left(G_{\| C}\right)$ was calculated by the following expression [30]:

$$
\mathrm{G}_{\mathrm{IIC}}=\frac{9 \mathrm{a}^{2} \mathrm{P} \delta}{2 \mathrm{~B}\left(2 \mathrm{~L}^{3}+3 a^{3}\right)}
$$

where $L$ is the support-span length: $48 \mathrm{~mm}$.

Stresses and strains were also calculated throughout the tests according to the international standard ASTM 7264 [31]. 


\section{Results and discussion}

In this section the fracture response of nanofiber-modified and reference laminates are presented.

Since 3 specimens have been tested for each configuration, the results are given by the mean value of each configuration.

\section{$D C B$ tests}

Figure 3-left illustrates examples of force-crack opening displacement (COD) curves of DCB tests for modified and reference samples. It is shown that the modified laminates can carry higher loads with respect to the pristine ones during almost all the tests. In particular the maximum force is almost $40 \%$ higher (Table 1 ) and the $G_{I C}$ of nanomodified samples increased about $62 \%$ with respect the virgin specimens.
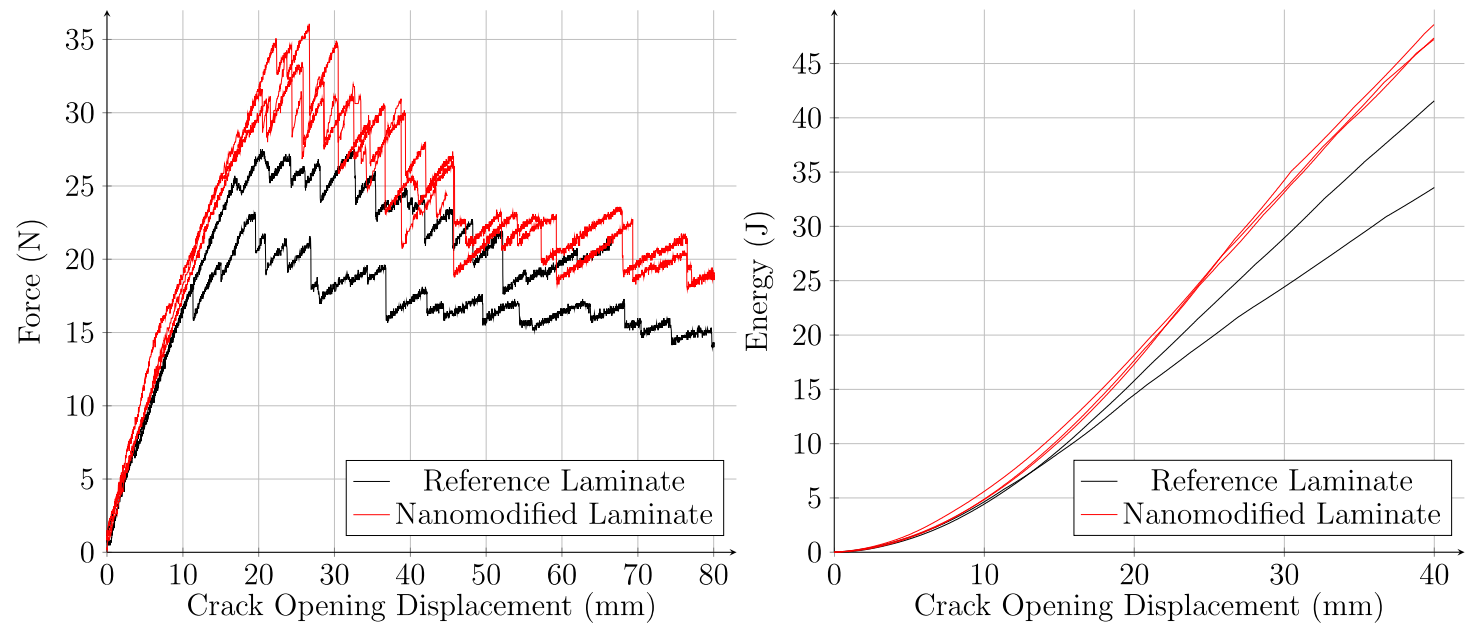

Figure 3. DCB fracture tests curves: left) Force vs. COD; right) Mechanical energy vs. COD.

Figure 3-right shows the mechanical energy dissipated during the tests until a COD of $40 \mathrm{~mm}$, by integration of the load-displacement curves.

Due to some problems while testing the reference laminates, one specimen became unusable, and for this reason Figure 3-left shows only two reference laminate curves. The authors chose not to 
re-manufacture the specimen because all the other specimens came from the same batch, and we wanted to make the test as fair a comparison as possible.

Table 1. Mode I fracture test results. $\mu$ and $\sigma$ are mean value and standard deviation respectively.

\begin{tabular}{|c|c|c|c|c|c|c|}
\hline & \multirow{3}{*}{\multicolumn{2}{|c|}{$\begin{array}{l}\text { Maximum load } \\
\text { (N) }\end{array}$}} & \multirow{3}{*}{\multicolumn{2}{|c|}{$\begin{array}{c}\text { Mechanical energy } \\
\text { @ } 65 \text { mm (J) }\end{array}$}} & \multirow{3}{*}{\multicolumn{2}{|c|}{$\begin{array}{c}\mathrm{G}_{\mathrm{IC}} \\
\left(\mathrm{kJ} / \mathrm{m}^{2}\right)\end{array}$}} \\
\hline & & & & & & \\
\hline & & & & & & \\
\hline & $\mu$ & $\sigma$ & $\mu$ & $\sigma$ & $\mu$ & $\sigma$ \\
\hline Virgin & 25.1 & 3.0 & 1.21 & 0.15 & 0.55 & 0.01 \\
\hline Nano-modified & 35.0 & 1.7 & 1.52 & 0.01 & 0.89 & 0.11 \\
\hline Gain (\%) & \multicolumn{2}{|c|}{$+39 \%$} & \multicolumn{2}{|c|}{$+25 \%$} & \multicolumn{2}{|c|}{$+62 \%$} \\
\hline
\end{tabular}

Table 1 presents fracture parameters for both the configurations. Up to a COD of $10 \mathrm{~mm}$, trends of the reference and nanomodified laminates are almost the same; after this point, nanomodified specimens absorb a greater amount of energy and as the tests go on the difference among the curves increases.

The curves clearly show that the nanomodified interfaces exhibit better properties than the virgin ones, and in particular, increments of $39 \%, 25 \%$, and $62 \%$ are registered for maximum load, maximum energy (after $65 \mathrm{~mm}$ of displacement), and $\mathrm{G}_{\mathrm{IC}}$, respectively.

\section{ENF tests}

Figure 4-left shows the stress-strain relation of Mode II fracture tests for pristine and nanomodified laminates. 

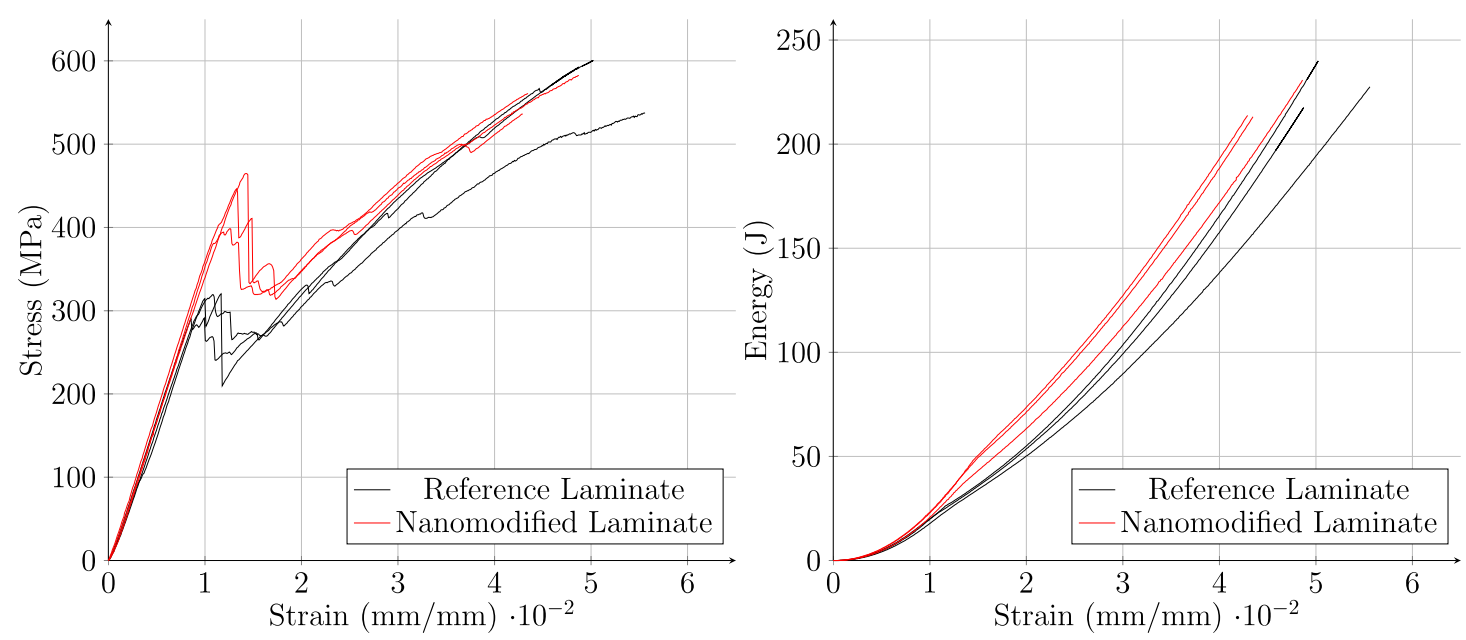

Figure 4. ENF fracture tests curves: left) Stress vs. Strain; right) Mechanical energy vs. Strain.

It is shown that modified laminates carry a significantly higher amount of load with respect to the reference specimen throughout the entire test. Table 2 presents the ENF results and shows that maximum stress is increased $41 \%$ by applying Nylon 6,6 nanofibers in the delaminated interface.

Table 2. Mode II fracture test results. $\mu$ and $\sigma$ are mean value and standard deviation respectively.

\begin{tabular}{|c|c|c|c|c|c|c|}
\hline & \multirow{2}{*}{\multicolumn{2}{|c|}{ Maximum stress }} & \multirow{2}{*}{\multicolumn{2}{|c|}{ Mechanical energy }} & \multirow{2}{*}{\multicolumn{2}{|c|}{$\mathbf{G}_{\| C}$}} \\
\hline & & & & & & \\
\hline & \multicolumn{2}{|c|}{ (MPa) } & \multicolumn{2}{|c|}{$@ 0.043$ mm/mm (J) } & \multicolumn{2}{|c|}{$\left(\mathrm{kJ} / \mathrm{m}^{2}\right)$} \\
\hline & $\mu$ & $\sigma$ & $\mu$ & $\sigma$ & $\mu$ & $\sigma$ \\
\hline Reference & 304 & 23 & 3.02 & 0.40 & 0.139 & 0.020 \\
\hline Nano-modified & 429 & 36 & 4.13 & 0.51 & 0.291 & 0.050 \\
\hline Gain (\%) & \multicolumn{2}{|c|}{$+41 \%$} & \multicolumn{2}{|c|}{$+37 \%$} & \multicolumn{2}{|c|}{$+109 \%$} \\
\hline
\end{tabular}

At the same time the critical strain energy release rate $G_{\| I}$ is more than doubled. Experiments show that the presence of nanofibers increases the load that the specimen can carry and at the same time reduces the crack propagation after failure. The trend of mechanical energy absorbed 
during the tests is shown in Figure 4-right. Energy has been calculated up to a common strain deformation of $0.043 \mathrm{~mm} / \mathrm{mm}$.

Maximum stress, mechanical energy, and $G_{\text {IIC }}$ of Nylon 6,6-nanomodified specimens resulted in increases of $41 \%, 37 \%$ and $109 \%$ respectively compared to virgin specimens, at the end of the test. Comparing the results of Mode I and Mode II fracture tests, presented in Tables 1 and 2, it is shown that the effect of Nylon 6,6 nanointerleave is more significant in Mode II loading than in Mode I, especially for initial energy release rates; $G_{\| I}$ is increased about the double of $G_{\mid C}$.

\section{Fracture surface morphology}

In a previous work of some of the authors [24] it is shown that a nanofibrous mat interleaved between two layers of a laminate has the capability to bridge the two plies even when the matrix is broken, carrying on additional loads. This is one of the most important reinforcing effects that a nanointerleave brings to the laminate. In Figure 5 SEM micrographs of fractured interfaces of virgin (Figures 5-A and 5-C) and nanomodified (Figures 5-B and 5-D) specimens are shown. Figures 5-A and 5-B are taken from DCB specimens, while Figures 5-C and 5-D show fracture surfaces of ENF samples. 

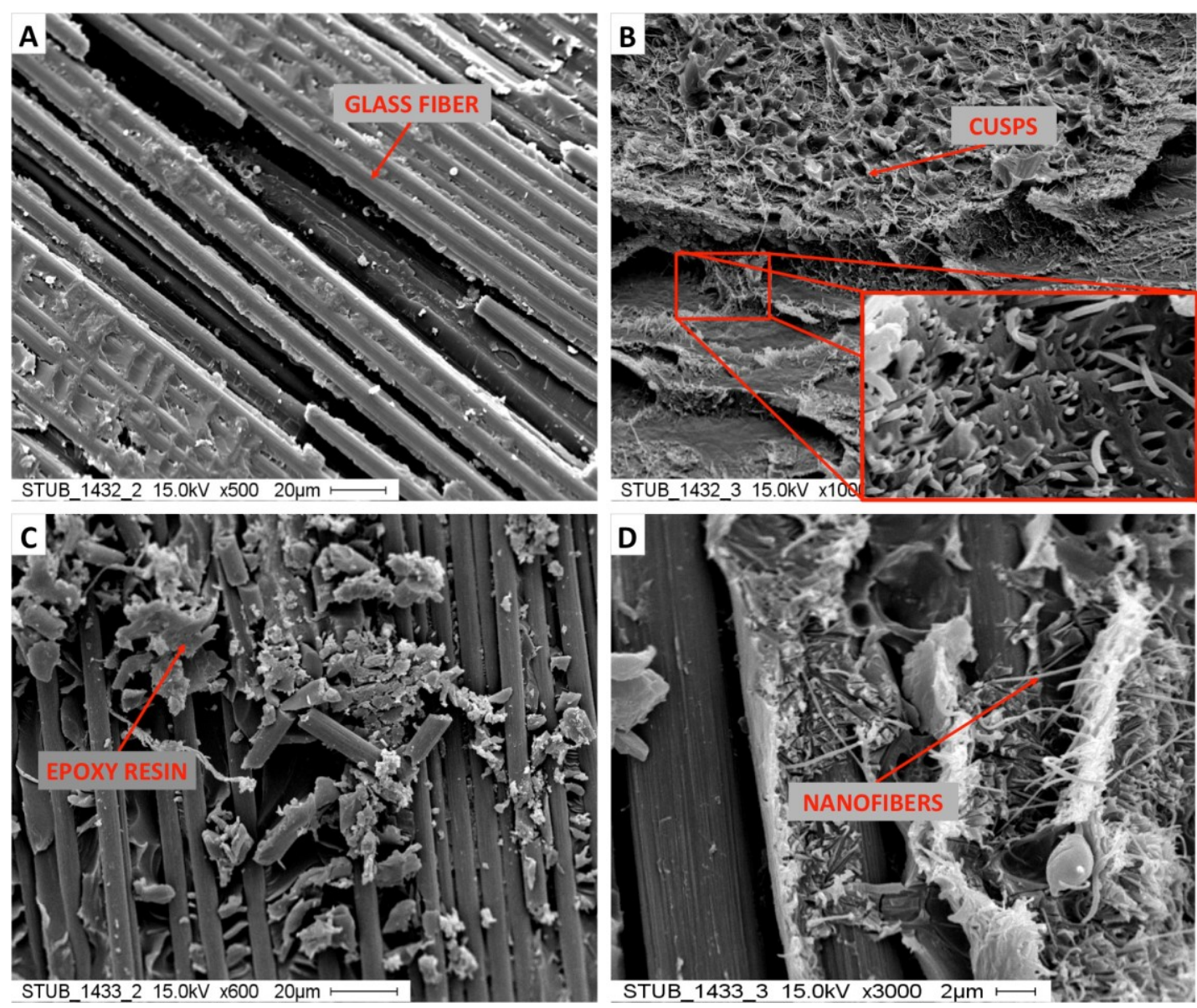

Figure 5. SEM micrographs of fractured surfaces from DCB (A-B) and ENF (C-D) tests, Virgin (A-C) and Nanomodified (B-D) interfaces.

As shown by Greenhalgh [32] in both the virgin and nanomodified cases, the fracture surfaces of Mode II specimens present a higher roughness due to the formation of cusps while the surfaces slide one over the other. Furthermore nanofibers interleaved into interfaces cause a significant increase of quantity of cusps, leading to a higher energy to break the surface. From images taken of Mode I specimens, it can be noted that the fracture surface of nanomodified samples (see Figures 5-B and 5-D) is more complex than the non-modified ones (see Figures $5-A$ and $5-C$ ). Glass fibers from virgin interfaces were found to be smooth and continuous, while the nanomodified interfaces appear much rougher. It is possible to observe that the nanofibers coming out from the delaminated interfaces (see Figure 5-B) still link the two layers they are inserted between, even when the matrix around them is broken (see Figure 5-D). While the crack propagates during the 
test, nanofibers help the plies to remain bonded together and hinder the crack propagation. Due to the higher ductility of the Nylon 6,6 compared with the resin, when the epoxy fails due to excessive strain, nanofibers act as a fiber bridging mechanism to link the two plies, giving an additional amount of resistance to the interface.

Experiments presented in the present study and in previous work of some of the authors [23] highlight that the nanoreinforcing effect is more significant for Mode II loading rather than Mode I. Numerical simulations presented in $[33,34]$ show that at any instant of time, the amount of nanofibers involved in the loading process is much higher during a Mode II test than during a Mode I, significantly increasing the reinforcement effect. It is due to the load direction: during Mode I tests, the two interfaces are opened and the stress is concentrated on the crack tip, involving a small portion of material and thus of nanofibers. As the crack propagates, the distance between the interfaces grows bigger than the nanofibers length and the nanoreinforce breaks too. Under Mode II, the two parts of the specimens slide only for few millimetres, allowing the nanofibers to carry the load for a longer time after the crack is propagated and the amount of reinforce working at each instant is much higher than during the DCB.

The presence of nanofibers encourages the phenomenon of migration introduced in [32] which inhibits fracture propagation. Nanofibers force the crack tip to follow a longer path, even if in the same interface with respect that followed in virgin specimens, requiring a higher energy to propagate.

\section{Conclusions}

The effect of Nylon 6,6 nanofibrous interleave on Mode I and Mode II fracture mechanics behaviour of glass fiber/epoxy resin composite laminate is here investigated. Nylon nanofiber mats were prepared by electrospinning and then interleaved in the mid-plane of laminate 
specimens for DCB and ENF tests to investigate Mode I and Mode II fracture mechanics of virgin and nanomodified specimens.

SEM pictures of the fractured interfaces have been used to explain the crack propagation mechanism and the role of the nanofibers into the interface. The main findings are summarized as follows:

1. Nylon 6,6 nanofibers improve the fracture behaviour of laminates under both Mode I and Mode II fracture mechanics loads;

2. experiments show that nanomodified interfaces exhibit better mechanical characteristics than virgin interfaces in terms of maximum load, energy absorption and critical strain energy release rate. It is also shown that the effectiveness of the nanoreinforce depends on the load direction: interfaces loaded under Mode II register a significant higher improvement than those loaded under Mode I. In particular maximum stress, mechanical energy, and $G_{\| C}$ of nanomodified specimens are increased of $39 \%, 25 \%$, and $62 \%$ respectively for Mode I loads, and 41\%, 37\% and 109\% respectively for Mode II, compared to pristine specimens;

3. curing process has a very important role in fracture mechanic's improvement of nanointerleaved specimens. By keeping the specimens into the autoclave, under pressure, during the night, the mixture of epoxy and Nylon 6,6 nanofibers significantly improved compared to traditional interleaving-and-curing processes.

4. SEM micrographs show that due to the higher ductility of the Nylon 6,6 compared to the resin, when the matrix fails, nanofibers are still able to bridge the plies, even after the breakage of the epoxy. Fractured surface morphology showed that nanomodified laminates have a significantly rougher surface compared to virgin ones. Nanofibers are able to inhibits the delamination propagation, forcing the crack to follow a longer path, and thus an higher amount of energy is required to let the crack propagates; 


\section{Supplementary materials}

Underlying materials related to this research are owned by University of Bologna. However, if funders or other researchers want a copy of the data, they can make a request to the author.

\section{References}

1. Sridharan S. Delamination behaviour of composites. Cambridge: CRC Press, 2008.

2. Marieta C, Schulz E, Irusta L, Gabilondo N, Tercjak A and Mondragon I. Evaluation of fiber surface treatment and toughening of thermoset matrix on the interfacial behaviour of carbon fiber-reinforced cyanate matrix composites. Compos Sci Technol 2005; 65: 2189-2197.

3. Hojo M, Matsuda S, Tanaka M, Ochiai S and Murakami A. Mode I delamination fatigue properties of interlayer-toughened CF/epoxy laminates. Compos Sci Technol 2006; 66: 665-675.

4. Yang $\mathrm{T}$, Wang $\mathrm{CH}$, Zhang J, He S and Mouritz AP. Toughening and self-healing of epoxy matrix laminates using mendable polymer stitching. Compos Sci Technol 2012; 72: 1396-1401.

5. Fuoss E, Straznicky PV and Poon C. Effects of stacking sequence on the impact resistance in composite laminates - Part 1: parametric study. Compos Struct 1998; 41: 67-77.

6. Howard WE, Gossard T, Jones RM and Robert MJ. Composite laminate free-edge reinforcement with U-Shaped Caps. Part I: stress analysis. AIAA Jour 1986; 27: 610-616.

7. Partridge IK and Cartie DDR. Delamination resistant laminates by Z-Fiber pinning: Part I manufacture and fracture performance. Compos Part A-App/ 2005; 36: 55-64.

8. Tang G, Yan Y, Chen X, Zhang J, Xu B and Feng Z. Dynamic damage and fracture mechanism of three-dimensional braided carbon fiber/epoxy resin composites. Mater Design 2000, 22, 2125. 
9. Zucchelli A, Focarete ML, Gualandi C and Ramakrishna S. Electrospun nanofibers for enhancing structural performance of composite materials. Polym Adv Technol 2011; 22: 339349.

10. Dzenis YA and Reneker DH. Delamination resistant composites prepared by small diameter fiber reinforcement at ply interfaces. Patent US 6265333, USA, 20112001.

11. Palazzetti R, Zucchelli A, Gualandi C, Focarete ML, Donati L, Minak G and Ramakrishna S. Influence of electrospun Nylon 6,6 nanofibrous mats on the interlaminar properties of Gr-Epoxy composite laminates. Compos Struct 2012; 94: 571-579.

12. Liu L, Huang ZM, He CL and Han XJ. Mechanical performance of laminated composites incorporated with nanofibrous membranes. Mater Sci Eng A-Struct 2006; 435-436: 309-317.

13. Crane RM and Camponeschi ET. Experimental and analytical characterization of multidimensionally braided graphite/epoxy composites. Exp Mech 1986; 26: 259-266.

14. Steeves CA and Fleck NA. In-plane properties of composite laminates with throughthickness pin reinforcement. Int J Solids Struct 2006; 43: 3197-3121.

15. Chang P, Mouritz AP and Cox BN. Flexural properties of z-pinned laminates. Compos Part AApp/ 2007; 38: 244-251.

16. Macander Jr AB, Crane RM and Camponeschi ET. Fabrication and Mechanical Properties of Multidirectionally Braided Composite Materials. ASTM STP 893, 1986: 422.

17. Zhang J, Yang $\mathrm{T}$, Lin $\mathrm{T}$ and Wang $\mathrm{CH}$. Phase morphology of nanofibre interlayers: critical factor for toughening carbon/epoxy composites. Compos Sci Technol 2012; 72: 256-262.

18. H. Saghafi, T. Brugo, G. Minak, A. Zucchelli, The effect of PVDF nanofibers on mode-I fracture toughness of composite materials, Compos Part B-Eng 2015; 72: 213-216.

19. Zhang J, Lin T and Wang X. Electrospun nanofibre toughened carbon/epoxy composites: effects of polyetherketone cardo (PEK-C) nanofibre diameter and interlayer thickness. Compos Sci Technol 2010; 70: 1660-1666. 
20. Li G, Li P, Zhang C, Yu Y, Liu H, Zhang S, Jia X, Yang X, Xue Z and Ryu S. Inhomogeneous toughening of carbon fiber/epoxy composite using electrospun polysulfone nanofibrous membranes by in situ phase separation. Compos Sci Technol 2008; 68: 987-994.

21. Magniez K, Chaffraix T and Fox B. Toughening of a carbon-fibre composite using electrospun poly(hydroxyether of bisphenol A) nanofibrous membranes through inverse phase separation and inter-domain etherification. Materials 2011; 4: 1967-1984.

22. Shivakumar K, Lingaiah S, Chen H, Akangah P, Swaminathan G and Russell L. Polymer nanofabric interleaved composite laminates. AIAA Journal 2009; 47: 1723-1729.

23. Palazzetti R, Yan XT and Zucchelli A. Influence of geometrical features of electrospun nylon 6,6 interleave on the CFRP laminates mechanical properties. Polym Compos 2014; 35: 137-150.

24. Palazzetti R, Zucchelli A and Trendafilova I. The self-reinforcing effect of Nylon 6,6 nanofibres on CFRP laminates subjected to low velocity impact. Compos Struct 2013; 106: 661-671.

25. Sadeghian R, Gangireddy S, Minaie B and Hsiao KT. Manufacturing carbon nanofibers toughened polyester/glass fiber composites using vacuum assisted resin transfer molding for enhancing the mode-I delamination resistance. Compos Part A-App/ 2006; 37: 1787-1795.

26. Saghafi H, Zucchelli A, Palazzetti R and Minak G. The effect of interleaved composite nanofibrous mats on delamination behaviour of polymeric composite materials. Compos Struct 2014; 109: 41-47.

27. Palazzetti R. Flexural behaviour of carbon and glass fiber composite laminates reinforced with Nylon 6,6 electrospun nanofibers. J Comp Mat, In press, DOI: 10.1177/0021998314565410 28. Alessi, S Di Filippo M, Dispenza C, Focarete, ML, Gualandi C, Palazzetti R, Pitarresi G and Zucchelli A. Effects of Nylon 6,6 Nanofibrous Mats on Thermal Properties and Delamination Behavior of High Performance CFRP Laminates. Pol Comp 2014, In press, DOI: 10.1002/pc 29. ASTM D5528 2008. Standard Test Method for Mode I Interlaminar Fracture Toughness of Unidirectional Fiber-Reinforced Polymer Matrix Composites. 
30. Protocol No 2 for interlaminar fracture toughness testing of composites: Mode II. European Structural Integrity Society, 1993.

31. ASTM D7264 2007. Standard Test Method for Flexural Properties of Polymer Matrix Composite Materials.

32. Greenhalgh ES, Rogers C and Robinson P. Fractographic observations on delamination growth and the subsequent migration through the laminate. Compos Sci Technol 2009; 69: $2345-2351$.

33. Moroni F, Palazzetti R, Zucchelli A and Pirondi A. A numerical investigation on the interlaminar strength of nanomodified composite interfaces. Compos Part B-Eng 2013; 55: 635641.

34. Giuliese G, Palazzetti R, Moroni F, Zucchelli A and Pirondi A. Cohesive zone modelling of delamination response of a composite laminate with interleaved nylon 6,6 nanofibers. Compos Part B-Eng 2015; DOI: 10.1016/j.compositesb.2015.03.087 